\title{
YES! OXIDATIVE STRESS MAY BE A RISK FACTOR FOR CHRONIC PERIODONTITIS PATIENTS
}

\begin{tabular}{ll} 
Dr. Archith Mannan & $\begin{array}{l}\text { Post Graduate Student, Department Of Periodontology, Sri Sankara Dental } \\
\text { College, Varkala, Kerala-695318. }\end{array}$ \\
\hline Dr Mathew John* & $\begin{array}{l}\text { Professor And HOD, Department Of Periodontology, Sri Sankara Dental } \\
\text { College, Varkala, Kerala-695318. }{ }^{*} \text { Corresponding Author }\end{array}$ \\
\hline $\begin{array}{l}\text { Dr. Bindu Rachel } \\
\text { Thomas }\end{array}$ & $\begin{array}{l}\text { Professor, Department Of Periodontology, Sri Sankara Dental College, } \\
\text { Varkala, Kerala-695318. }\end{array}$ \\
\hline $\begin{array}{l}\text { Dr. Sabari } \\
\text { Chandramohan }\end{array}$ & $\begin{array}{l}\text { Associate Professor, Department Of Periodontology, Sri Sankara Dental } \\
\text { College, Varkala, Kerala-695318. }\end{array}$ \\
\hline Dr. Aswathy S. & $\begin{array}{l}\text { Assistant Professor, Department Of Periodontology, Sri Sankara Dental } \\
\text { College, Varkala, Kerala-695318. }\end{array}$ \\
\hline Dr. Revu Das S. D. & $\begin{array}{l}\text { Assistant Professor, Department Of Periodontology, Sri Sankara Dental } \\
\text { College, Varkala, Kerala-695318. }\end{array}$ \\
\hline \hline
\end{tabular}

ABSTRACT Oxidative stress is defined as the condition occurring when the physiological balance between oxidants and antioxidants is disrupted in favor of the former with potential damage for the organism. The pathological events leading to the destruction of the periodontium encompasses complex interactions involving an imbalance between oxidant and antioxidant enzymatic and non-enzymatic defense mechanisms. Periodontitis is an oral inflammatory disorder affecting alveolar bone, gingiva, and periodontal ligament initiated by the complex interaction between the presence of microorganisms (periodontopathic bacteria) and the host's immune response. Whole saliva samples were collected from 25 chronic periodontitis patients. Saliva samples were centrifuged and the supernatant was used to determine the 8-OHdG levels with a competitive ELISA kit. To determine the clinical condition of each subject, the Bleeding on Probing (BOP), Clinical attachment level (CAL), and Pocket probing depth (PPD) were measured. This study shows significant correlation between increased values of oxidative stress biomarker and clinical parameters in chronic periodontitis patients.

\section{KEYWORDS : Periodontitis, Oxidative Stress, 8-OHdG}

\section{INTRODUCTION}

Humans survive in an oxygen rich environment, and their survival depends on a subtle mitochondrial current that generates ATP, the common energy source for all cells. Electrons, however can escape from the mitochondrial transport chain and generate reactive oxygen species [ROS], such as hydrogen peroxide $\left[\mathrm{H}_{2} \mathrm{O}_{2}\right]$, oxygen superoxide $\left[\mathrm{O}_{2}\right]$ or the hydroxyl radical $[\mathrm{OH}]$. These are highly reactive oxygen radicals that are liable for most oxidative stress on cells. Oxidative stress, occurring as a consequence of imbalance between the oxidant actors and protective antioxidant defense system. Oxidative stress has been implicated as a significant contributor in over 100 disorders and more recently periodontitis

Several studies have demonstrated that $8-\mathrm{OHdG}$ in bodily fluids can act as a biomarker of oxidative stress [1],[2] and 8OHdG is commonly used as a marker to evaluate oxidative DNA damage in disorders including chronic inflammatory diseases.

Oxidative stress is induced by an imbalance between excessive ROS production and anti-oxidant mechanisms. Increased levels of ROS leading to a state of Oxidative Stress have been implicated in the pathogenesis of a large number of diseases, including cardiovascular diseases and diabetes [3].

A study done by Chapple et al in 2007 reported that oxidative Stress has been linked with both onset of periodontal tissue destruction $^{4}$ and systemic inflammation [5].

In the present study we hypothesized that the level of 8-OHdG in saliva increases by the periodontal destruction severity determined by clinical parameters as clinical attachment level, Probing pocket depth, Bleeding on Probing.

\section{SUBJECTS AND METHODS}

\section{Experimental design}

This observational analytical study was carried out between june-december, 2019 in (Department of Periodontics, Sri Sankara dental college, Varkala). Twenty five subjects were included in the study with inclusion criteria ; Patients with moderate/severe chronic periodontitis, Subjects having $\geq$ $30 \%$ sites with CAL $3 \mathrm{~mm}$, at least $30 \%$ sites in with $\mathrm{PD} \geq 5 \mathrm{~mm}$ and bleeding on probing ,age between $30-50$ years, having at least 20 teeth excluding third molars, radiographic evidence of generalized bone loss, willing to give an informed consent .

Exclusion criteria for the study were history of periodontal therapy in the previous 6 months ,history of alcoholism and smoking, anti-inflammatory, antimicrobial and immunosuppressive therapies during previous 6 months, Systemic conditions that could affect the progression of periodontitis (e.g. diabetes, immunologic disorders), pregnant, lactating, postmenopausal subjects Needing antibiotics for infective endocarditis prophylaxis during dental procedures; having acute illness symptoms (i.e., fever, sore throat, body aches, or Diarrhoea).

\subsection{Sample collection}

Unstimulated whole saliva $(2 \mathrm{~mL})$ was collected in the morning (10am-12 pm) from all the subjects. All the subjects were asked to refrain from eating, drinking and physical exercise for a minimum of two hours before saliva collection. Subjects were comfortably seated and asked to rinse their mouth using distilled water. After a few minutes of relaxation, they were asked to first swallow saliva and after 5 minutes remaining saliva in the mouth was spit into the sterile container collected saliva was placed in a nitrogen flask till transport to the lab, where it was stored at $-80^{\circ} \mathrm{C}$. Before analysis, samples were defrosted, centrifuged at $12,000 \mathrm{rpm}$ for $10 \mathrm{~min}$ in a centrifuge 
to remove the particulates and the supernatant was separated and analyzed using an ELISA test.

\subsection{Assessment Of clinical Parameters}

Probing pocket depth (PPD), clinical attachment loss (CAL) were recorded immediately before saliva collection.

I. Probing pocket depth (PPD): All teeth will be probed at six sites (mesiobuccal, midbuccal, distobuccal, mesiolingual, midlingual, distolingual) using Williams Graduated periodontal probe, to the nearest millimeter. It is measured from crest of marginal gingival to the depth of the sulcus. For diagnosis of generalized moderate to severe chronic periodontitis $\geq 30 \%$ sites with $P D \geq 5 \mathrm{~mm}$.

II. Bleeding on Probing (BOP): Full mouth bleeding on probing will be registered in conjunction with periodontal probing after 30 seconds. The bleeding will be recorded as present (1) or absent (0).

III. Clinical Attachment Loss (CAL): It is measured from CEJ to the base of the sulcus using Williams Graduated Periodontal Probe, to the nearest millimeter. Individuals with periodontal disease should have $\geq 30 \%$ of sites with CAL at least $3 \mathrm{~mm}$.

\subsection{Elisa Assay Procedure}

Before adding saliva samples to wells, equilibrated the TMB substrate for at least $30 \mathrm{~min}$ at room temperature $\left(37^{\circ} \mathrm{C}\right)$. When diluting samples and reagents, they must be mixed completely and evenly. It was recommend to plot a standard curve for each test. Set standard, test sample and control (zero) wells on the pre-coated plate respectively, and then, recorded their positions. Washed the plate 2 times before adding standard, sample and control (zero) wells then 50 L of Standard, Blank, or Sample were added per well. The blank well was added with Sample/Standard dilution buffer. Immediately $50 \mathrm{~L}$ of Biotin-detection antibody working solution was added to each well and covered with the Plate sealer provided and gently tapped the plate to ensure thorough mixing, incubated for 45 minutes at $37^{\circ} \mathrm{C}$, aspirated and washed 3 times by filling each well with Wash Buffer (approximately $350 \mathrm{~L}$ ). Then $100 \mathrm{~L}$ of SABC working solution was added to each well again covered with a new Plate sealer and incubated for 30 minutes at $37^{\circ} \mathrm{C}$. Then repeatedly done the aspiration/wash process for five times and $90 \mathrm{~L}$ of TMB Substrate was added to each well, covered with a new Plate sealer, incubated for about $15-20$ minutes at $37^{\circ} \mathrm{C}$. When apparent gradient appeared in standard wells, terminated the reaction and added $50 \mathrm{~L}$ of Stop Solution to each well, the color turned to yellow immediately. Then stop solution was added, adding order of stop solution should be as the same as the substrate solution. Finally, determined the optical density (OD Value) of each well at once, using a microplate reader set to $450 \mathrm{~nm}$, the concentration of the corresponding sample was calculated.

\section{STATISTICAL ANALYSIS}

The correlations between saliva 8-OHdG levels and clinical parameters were calculated using Pearson's correlation test in this study, where the value $r=1$ means a perfect positive correlation and the value $r=-1$ means a perfect negative correlation. A p value $\leq 0.001$ was considered statistically significant.

\section{RESULTS}

A total of 25subjects were included in the study based on inclusion and exclusion criteria. 8 females and 17 males were included in the study with general chronic periodontitis (AAP 1999).

The study showed significant increase in levels of 8- OHdG salivary biomarker as the severity of periodontal disease progress. The mean probing pocket depth in patients with chronic periodontitis were statistically significant in relation to salivary 8-OHdG biomarker $(\mathrm{p}<0.001)$ (Table 1).Between mean clinical attachment level in patients with chronic periodontitis and salivary $8-\mathrm{OHdG}$ biomarker showed statistically significant $(p<0.001)$ (Table 2$)$

A strong positive correlation between mean probing pocket depth with salivary 8-OHdG levels was observed (graph 1). Significant increase in mean clinical attachment level with salivary 8-OHdG levels was also noted in the study (graph 2)

The mean salivary 8-OHdG level in 25 subjects with chronic periodontitis was $4.28 \pm 0.1 \mathrm{ng} / \mathrm{ml}$.

Table 1: Correlation Between mean probing pocket depth and salivary 8-OHdG biomarker

\begin{tabular}{|c|c|c|c|}
\hline Mean PPD & $\mathbf{n}$ & $\mathbf{r}$ & $\mathbf{p}$ \\
\hline 7.952 & 25 & 0.8228 & $\mathrm{P}<0.01$ \\
\hline
\end{tabular}

(PPD: probing pocket depth, n: number of subjects, $r$ : Pearson's correlation coefficient, P: probability value) **:- Significant at 0.01 level

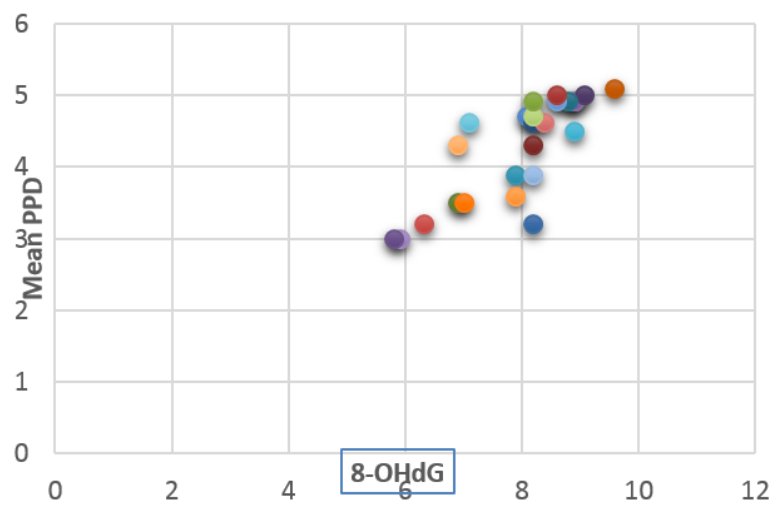

Graph 1: Scatter diagram for mean probing pocket depth and salivary 8-OHdG levels

Table 2: Correlation Between mean clinical attachment level and salivary 8-OHd biomarker

\begin{tabular}{|c|c|c|c|}
\hline Mean CAL & $\mathbf{n}$ & $\mathbf{r}$ & $\mathbf{p}$ \\
\hline 8.536 & 25 & 0.7664 & $\mathrm{P}<0.01$ \\
\hline
\end{tabular}

(CAL: clinical attachment level, $n$ : number of subjects, $r$ : Pearson's correlation coefficient, P: probability value) **:Significant at 0.01 level

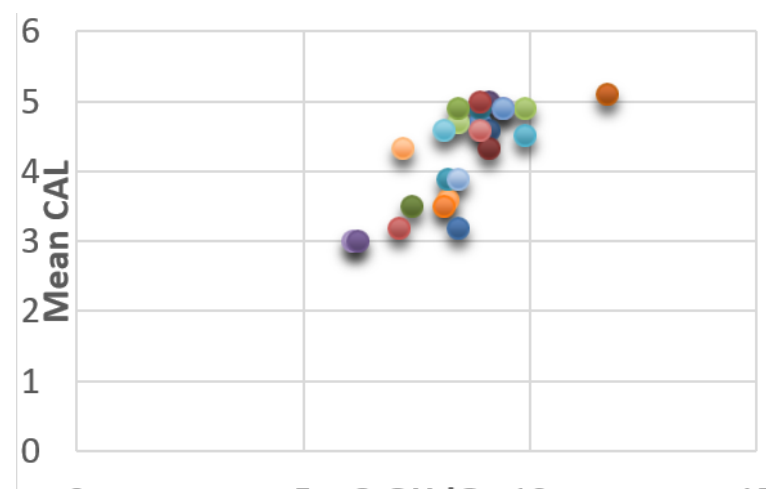

\section{0}

5 8-OHdG 10

15

Graph 2: Scatter diagram for mean clinical attachment level and salivary 8-OHdG level

\section{DISCUSSION}

Periodontal diseases are one of the most widespread chronic conditions affecting the larger populations of the world. Periodontal disease is initiated by the colonization of bacterial pathogens, such as Porphyromonas gingivalis, Aggregatibacter actinomycetemcomitans and Tannerella 
forsythus. These microorganisms have the capacity to stimulate the host defense mechanisms to produce reactive oxygen species that damage the nearby host tissue in addition to destroying the pathogens [6].

Oxidative stress, is an imbalance between free oxygen radicals and antioxidant defense system, and is capable of causing damage to various cellular and extracellular components.8-OHdG is one of the major products of nucleotide oxidation in DNA. Salivary 8- OHdG levels are often studied in oral pathology, including periodontitis and oral cancers. It was found that salivary 8-OHdG levels were very high in patients with periodontally hopeless teeth and teeth with advanced periodontal breakdown.

Saliva is a fluid that is easily available and contains locally produced microbial and host response mediators that may offer the basis for a patient spesific diagnostic test for periodontitis. It includes constituents of non-salivary origin derived from gingival crevicular fluid (GCF), expectorated bronchial secretions, serum, blood cells from oral wounds, as well as bacteria and bacterial products, viruses and fungi, desquamated epithelial cells and food debris .

Oxidative damage to lipids, proteins, and nucleic acids can be monitored using established markers that are present in saliva. The indicators for oxidative stress are products obtained from oxidatively damaged macro-molecules. Proposed markers for periodontal disease include bacterial products, immunoglobulins and host enzymes [7]. Established salivary markers of oxidative stress include Malondialdehyde (MDA), thiobarbituric acid-reacting substances (TBARS) as a marker of lipid peroxidation, advanced oxidation protein products (AOPP) as a marker of protein oxidation, and advanced glycation end products (AGEs) as a marker of protein glycation and, thus, carbonyl stress that is tightly interconnected with oxidative stress.. After exposure of mammalian chromatin to a free radical, various DNA-base adducts have been identified. Of these, 8-OHdG is one of the most common stable products of oxidative DNA damage following specific enzymatic cleavage and acts as one of the most sensitive biomarkers for oxidative stress [8].

In this study, we first examined the oxidative DNA damage in periodontitis patients by measuring the salivary 8 -OHdG levels, and these levels were compared not only according to presence of disease but also but also according to disease severity. CAL which shows the disease severity may be affected by oxidative DNA damage, and as a result elevated salivary level of 8-OHdG, which shows disease activity, can be detected. This hypothesis coheres with the characteristic of chronic periodontitis.

Canakci et al. reported that high salivary 8-OHdGlevels and low antioxidant activity increased oxygen radical activity [9].

In another study reporting the correlation between 8-OHdG and periodontopathogens, it was suggested that the determination of salivary 8-OHdG was a useful biomarker to evaluate periodontal condition and effectiveness of periodontal therapy [10].

Dede et al. evaluated the effects of initial periodontal treatment on GCF and salivary levels of $8-\mathrm{OHdG}$ in periodontitis, and they revealed that 8-OHdG level in GCF was significantly decreased after initial periodontal therapy, whereas salivary levels of 8-OHdG did not change [11].

In a previous study undertaken by Takane et al, authors found significantly higher salivary 8-OHdG levels in subject with periodontally hopeless teeth (high level of attachment loss / severe periodontitis) than those in subject without periodontally hopeless teeth and those in clinically healthy controls [12].
All of these findings have supported that 8-OHdG levels in GCF and saliva reflect oxidative DNA damage in the presence of periodontal disease.

The present study suggests the involvement of oxidative stress in the periodontal environment which was reflected in the biological fluid is as a result of inflammatory reaction .Saliva can be easily collected, and therefore measurement of salivary 8-OHdG levels may prove to be useful in identifying patients at risk of tooth loss. Moreover, salivary analysis for periodontal diagnosis may prove a cost effective method for screening large populations

\section{CONCLUSION}

We suggest that elevated salivary levels of 8-OHdG may be a marker for disease activity and it reflects on directly disease severity parameters such as CAL, PD. However, further investigations are needed to clarify the exact mechanism of oxidative DNA damage in diseased periodontium.

\section{REFERENCES}

1. Chiou C, Chang P, Chan E, Wu T, Tsao K, Wu J. Urinary 8-hydroxydeoxyguanosine and its analogs as DNA marker of oxidative stress: development of an ELISA and measurement in both bladder and prostate cancers. ClinicaChimicaActa. 2003; 334:87-94.

2. H. Liu, M. Uno, K.T. Kitazato, A. Suzue, S. Manabe, H. Yamasaki, M. Shono and S. Nagahiro, Peripheral oxidative biomarkers constitutea valuable indicator of the severity of oxidative brain damage in acute cerebral infarction, Brain Res 29(1025) (2004), 43-50.

3. Castelao JE, Gago-Dominguez M (2008). Risk factors for cardiovascular disease in women: relationship to lipid peroxidation and oxidative stress. Med Hypotheses 79:31-44.

4. Chapple IL, Matthews JB (2007). The role of reactive oxygen and antioxidant species in periodontal tissue destruction. Periodontol 2000 43:160-232.

5. Basu S, Helmersson J, Jarosinska D, Sallsten G, Mazzolai B, Barregard $\mathrm{L}(2009)$. Regulatory factors of basal $\mathrm{F}(2)$-isoprostane formation: population age, gender and smoking habits in humans. Free Radic Res 43:85-91.

6. Kakimoto M, Inoguchi T Sonta T, et al. Accumulation of 8-hydroxy-2'deoxyguanosine and mitochondrial DNA deletion in kidney of diabetic rats. Diabetes 2002; 51:1588-1595.

7. Kaufman E and Lamster IB. Analysis of saliva for periodontal diagnosis--a review. Journal of Clinical Periodontology 2000; 27:453-465.

8. Kasai $\mathrm{H}$ and Nishimura $\mathrm{S}$. Hydroxylation of deoxyguanosine at the C-8 position by ascorbic acid and other reducing agents. Nucleic Acids Research 1984; 12:2137-2145

9. Canakci CF, Cicek Y, Yıldınm A, Sezer U, Canakci V. Increased levels of 8hydroxydeoxyguanosine and malondialdehyde and its relationship with antioxidant enzymes in saliva of periodontitis patients. Eur J Dent 2009;3:100106.

10. Sawamoto Y, Sugano N, Tanaka H, Ito K. Detection of periodontopathic bacteria and an oxidative stress marker in saliva from periodontitis patients. Oral Microbiol Immunol 2005;20:216-220.

11. Dede FO,Ozden FO,Avc1 B. 8-hydroxy-deoxyguanosine levels in gingival crevicular fluid and saliva in patients with chronic periodontitis after initial periodontal treatment.J Periodontol 2013;84:821-828.

12. M. Takane, N. Sugano, T. Ezawa, T. Uchiyama and K. Ito,A marker of oxidative stress in saliva: association with periodontally-involved teeth of a hopeless prognosis, J Oral Sci 47 (2005), 53-57. 